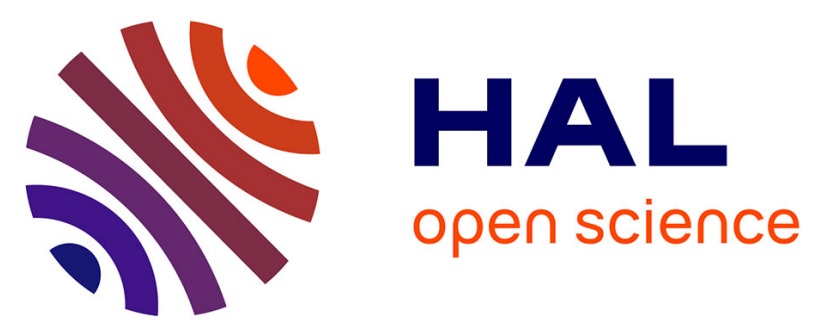

\title{
Design and fabrication of blazed binary diffractive elements with sampling periods smaller than the structural cutoff
}

Philippe Lalanne, Simion Astilean, Pierre Chavel, Edmond Cambril, Huguette Launois

\section{To cite this version:}

Philippe Lalanne, Simion Astilean, Pierre Chavel, Edmond Cambril, Huguette Launois. Design and fabrication of blazed binary diffractive elements with sampling periods smaller than the structural cutoff. Journal of the Optical Society of America. A Optics, Image Science, and Vision, 1999, 16 (5), pp.1143-1156. 10.1364/JOSAA.16.001143 . hal-00877423

\section{HAL Id: hal-00877423 \\ https://hal-iogs.archives-ouvertes.fr/hal-00877423}

Submitted on 28 Oct 2013

HAL is a multi-disciplinary open access archive for the deposit and dissemination of scientific research documents, whether they are published or not. The documents may come from teaching and research institutions in France or abroad, or from public or private research centers.
L'archive ouverte pluridisciplinaire HAL, est destinée au dépôt et à la diffusion de documents scientifiques de niveau recherche, publiés ou non, émanant des établissements d'enseignement et de recherche français ou étrangers, des laboratoires publics ou privés. 


\title{
Design and fabrication of blazed binary diffractive elements with sampling periods smaller than the structural cutoff
}

\author{
Philippe Lalanne, Simion Astilean, and Pierre Chavel \\ Laboratoire Charles Fabry de l'Institut d'Optique, Centre National de la Recherche Scientifique, \\ B.P. 147, F-91403 Orsay cedex, France
}

Edmond Cambril and Huguette Launois

Laboratoire de Microstructures et de Microélectronique, Centre National de la Recherche Scientifique, 196 avenue Henri Ravera, B.P. 107, F-92225 Bagneux, France

Received July 30, 1998; accepted December 17, 1998; revised manuscript received January 5, 1999

\begin{abstract}
We report here on the theoretical performance of blazed binary diffractive elements composed of pillars carefully arranged on a two-dimensional grid whose period is smaller than the structural cutoff. These diffractive elements operate under unpolarized light. For a given grating geometry, the structural cutoff is a period value above which the grating no longer behaves like a homogeneous thin film. Because the grid period is smaller than this value, effective-medium theories can be fully exploited for the design, and straightforward procedures are obtained. The theoretical performance of the blazed binary elements is investigated through electromagnetic theories. It is found that these elements substantially outperform standard blazed échelette diffractive elements in the resonance domain. The increase in efficiency is explained by a decrease of the shadowing effect and by an unexpected sampling effect. The theoretical analysis is confirmed by experimental evidence obtained for a $3 \lambda$-period prismlike grating operating at $633 \mathrm{~nm}$ and for a $20^{\circ}$-off-axis diffractive lens operating at $860 \mathrm{~nm}$. (C) 1999 Optical Society of America [S0740-3232(99)00105-2]

OCIS codes: $050.1380,050.1970,050.1950$.
\end{abstract}

\section{INTRODUCTION}

Diffractive optical elements have a variety of applications in (micro-) optical systems for beam shaping, deflecting, collimating, or imaging. The interest in diffractive optical elements is triggered by the availability of lithographic fabrication techniques. For the best performance, it is necessary to find optimum ways to synthesize and fabricate these elements. To this end, blazed diffractive elements that achieve a high diffraction efficiency in a specified order are required. Blazed diffractive elements can be fabricated either by a series of photolithography processes that approximate the surface relief with a multilevel structure ${ }^{1-4}$ or by direct-write technologies, such as single-point laser beam writing in photoresist, ${ }^{5}$ single-point diamond turning, ${ }^{6}$ or single-point electronbeam (e-beam) writing in polymers. ${ }^{7-9}$ In this paper we are concerned with the synthesis and the fabrication of diffractive elements composed of binary subwavelength pillars etched in a high-index material deposited on a glass substrate for visible-light operation. Their principle of operation relies on the analogy between periodic subwavelength-structured surfaces and artificial dielectric materials. In this analogy binary diffractive elements using just one photolithographic step simulate continuous phase delays through the effective-medium theory; for these blazed binary diffractive elements, the local fraction of matter removed is related to the local effective index. This approach is attractive because the fabrication relies only on lithographic technologies and etching techniques that are developed and continuously enhanced for the mass production of integrated circuits.

Artificial dielectric and metallic elements for control of surface reflection ${ }^{10}$ and for beam shaping ${ }^{11}$ were studied more than 30 years ago for operation in the microwave region of the spectrum. With the recent progress in nanofabrication technologies it was recently predicted ${ }^{12,13}$ that binary surface-relief diffractive elements, composed of subwavelength microstructures carefully arranged and etched in a transparent material, may be fabricated for visible-light operation. This possibility has received much attention from a modelization point of view. ${ }^{14-19}$ More importantly, it was successfully validated first in the thermal infrared ${ }^{20,21}$ and in the near-infrared ${ }^{15,22}$ regions and later on in the visible $e^{23-27}$ regions of the spectrum. With a few exceptions, ${ }^{23,24,27}$ the results of the above-mentioned studies ${ }^{12-27}$ hold for blazed binary diffractive elements with one-dimensional (1D) subwavelength features operating with linearly polarized light. This is probably because the effective-medium theory of two-dimensional (2D) subwavelength gratings is less understood than that of $1 \mathrm{D}$ gratings. We are concerned here with diffractive elements for operation with circular polarization or with unpolarized light.

Because of severe fabrication constraints, the first attempts in the visible region of the spectrum ${ }^{23-25}$ were not as successful as had been expected: The performance achieved is bad in comparison with that of diffractive elements fabricated with a continuous profile or with a 
multilevel-phase staircase profile. Only recently have encouraging results been obtained that offer experimentally better performance than that achieved theoretically by standard échelette gratings. ${ }^{26,27}$ This was made possible because a high-index material (namely, $\mathrm{TiO}_{2}$ ) was used to fabricate the blazed binary diffractive elements. In this way a drastic reduction in fabrication constraints is achieved. ${ }^{28}$ We also consider here blazed diffractive elements etched in a $\mathrm{TiO}_{2}$ layer deposited on a glass substrate.

In this paper the design, fabrication, and testing of blazed binary diffractive elements composed of pillars arranged on a $2 \mathrm{D}$ grid are considered. The grid period, called a sampling period hereafter, that is selected is smaller than or equal to the structural cutoff. For a given grating geometry, the structural cutoff is a period value intrinsic to the geometry considered (in the sense that it does not depend on the refractive indices of the substrate and the superstrate, for instance) above which the analogy between subwavelength dielectric gratings and homogeneous media ceases to be valid. ${ }^{27}$ It is denoted by $\Lambda_{s}$. The main results of this study cover three aspects:

1. In the resonance domain or, equivalently, for zone widths or grating periods equal to a few wavelengths, blazed binary diffractive elements are shown to substantially outperform conventional blazed diffractive elements with a continuous profile for operation with unpolarized light. Attempts to explain this enhanced efficiency are provided.

2. Straightforward procedures for designing highly efficient blazed binary diffractive elements in a simple, noniterative and nearly optimal way are proposed. These procedures do not rely on an extensive search for optimal performance by use of electromagnetic theory.

3. Experimental results show that, with current technology, blazed binary diffractive elements offering experimentally better performance than that achieved theoretically by conventional blazed diffractive elements are manufacturable for operation in the visible and nearinfrared regions of the spectrum.

In Section 2, gratings providing continuous phase delays are considered. By taking into account the shadowing effect due to the finite-element thickness, we argue that, in the resonance domain, graded-index gratings offer better performance than do standard échelette gratings and that one achieves higher performance by increasing the refractive index of the material patterned. Section 3 contains several general comments on the design and the fabrication of blazed binary diffractive elements. We first discuss the choice of the sampling period and emphasize, through illustrative examples, that better performance and simple designs are achieved for sampling periods smaller than or equal to the structural cutoff. We then describe the fabrication process that we used in the experimental part of this study and discuss its effect on the design of blazed binary diffractive elements. In Section 4, we focus on blazed binary gratings that deserve particular attention because of their major utility in optics. A specific design procedure aiming at lowering aspect-ratio requirements is proposed. The performance of this procedure is first studied theoretically and is then validated experimentally with a $3 \lambda$-period blazed binary grating operating at $633 \mathrm{~nm}$. In Section 5, blazed binary kinoforms are considered. Another design procedure is given. Its theoretical performance is investigated through electromagnetic theories for zone widths smaller than nine wavelengths. The theoretical predictions are supported by experimental evidence obtained for a $20^{\circ}$ off-axis diffractive lens operating at $860 \mathrm{~nm}$. Subsection 5.C reveals an unexpected sampling effect that is, in our opinion, the main reason that blazed binary diffractive elements substantially outperform conventional blazed diffractive elements with a continuous profile.

Throughout this paper and except otherwise mentioned, the numerical results provided for various grating geometries are all obtained for the following diffraction configuration: The incident medium is air (refractive index, 1); and the substrate is glass (refractive index, 1.52). An unpolarized plane wave (wavelength $\lambda$ in vacuum) is normally incident from air onto the diffractive element.

The analysis of (1D) gratings is performed by rigorous coupled-wave analysis ${ }^{29}$ and by its enhanced version (see Refs. 30 and 31). For the analysis of blazed gratings, the sawtooth profile is approximated by a stack of 15 lamellar gratings arranged in a staircase geometry. 2D gratings are analyzed with the new modal theory reported in Ref. 32. The computation of the effective index of $2 \mathrm{D}$ subwavelength gratings is performed with the plane-wave method along the lines set forth in Ref. 33, which incorporated the recent results on the Fourier analysis of discontinuous functions. ${ }^{32,34}$ In all cases good convergence is observed, and the numerical results provided hereafter can be considered as exact.

\section{BLAZED-INDEX GRATINGS AND ÉCHELETTE GRATINGS}

In the conventional design of thin phase elements the thin-element approximation is often applied, and the transmitted field behind the thin element is simply obtained by multiplication of the incident field with the transmission function of the thin element. For phase gratings the transmission function is directly related to the phase shifts that arise from propagation through the thin element. The phase shifts are obtained either by surface-relief elements or by gradient-index elements. Figure 1 illustrates our purpose for prismlike gratings. In Fig. 1(a), a standard blazed grating with a sawtooth profile is shown. The surface relief is assumed to be etched into a material of refractive index $n$. In Fig. 1(b), the equivalent graded-index element is shown; along the period the refractive index is linearly and continuously varying from 1 to a maximal value also denoted by $n$. In this case the grating is a periodic structure with a real graded index, and no subwavelength features mimicking artificial media are considered. Hereafter, the type of grating shown in Fig. 1(b) is called a blazed-index grating for differentiation with the type of grating shown in Fig. 1(a), which is simply called a blazed grating.

According to the thin-element approximation, blazed and blazed-index gratings have the same diffraction efficiency if Fresnel losses at the interfaces are neglected. 


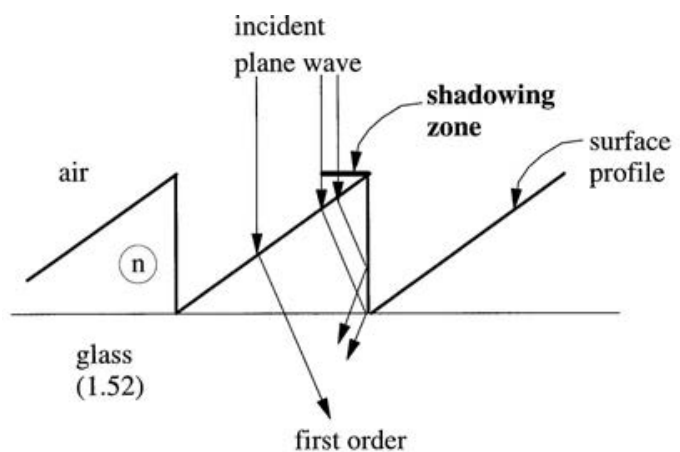

(a)

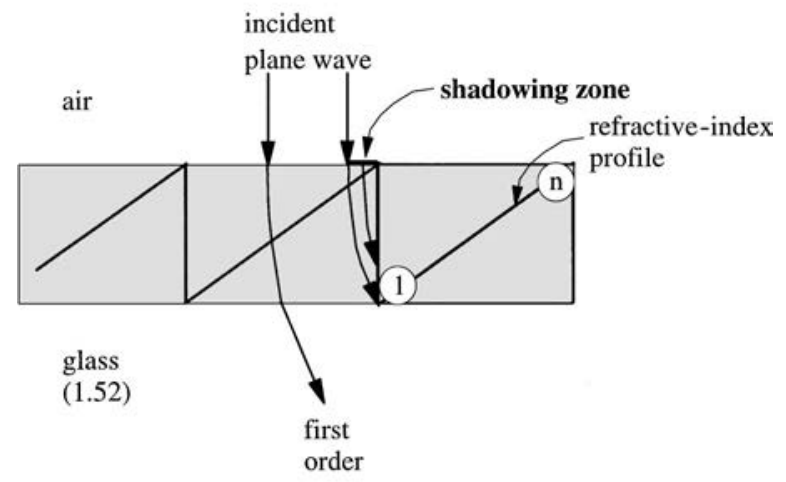

(b)

Fig. 1. (a) Blazed grating with a sawtooth échelette profile. (b) Blazed-index grating with a real graded index; along the period, the refractive index is linearly varying from 1 to $n$. The incident medium is air, the substrate is glass (refractive index, 1.52), and normal incidence from air is assumed. The concept of geometrically tracing rays through the finite depth of the gratings is used to sketch the light-shadowing effect.

The efficiency is $100 \%$ for a grating depth equal to $\lambda /(n$ - 1). For small period-to-wavelength ratios, the validity of the scalar diffraction theory for diffractive phase elements and the thin-element approximation cease to be valid, and electromagnetic theories have to be used for an accurate computation of the efficiency. ${ }^{35}$ This is illustrated in Fig. 2, where the first-order diffraction efficiency of blazed and blazed-index gratings is plotted for $n$ $=1.52$ as a function of the period-to-wavelength ratio. Results hold for unpolarized light and for a grating depth equal to $\lambda /(n-1)$. The solid and dotted curves correspond to blazed-index and blazed gratings, respectively. Even for a period as large as $8 \lambda$, the diffraction efficiency is significantly smaller than the scalar limit prediction of 96\% (100\% - 4\% because of Fresnel losses) obtained for blazed gratings. The drop in efficiency observed at small periods is well known and is considered to be a major obstacle for the production of high-speed and highperformance diffractive lenses. From Fig. 2 it is noteworthy that the blazed-index grating offers slightly better performance than the blazed grating. This difference in performance cannot be explained within the scope of the thin-element approximation but can be understood qualitatively if one takes into account some effects of the finite grating thickness through ray tracing. ${ }^{36}$ In the raytracing method, also known as the extended scalar theory by Swanson, ${ }^{37}$ the drop in efficiency observed at small periods is explained by a light-shadowing effect. As illus- trated in Fig. 1, the shadowing zone is determined by simple geometrical considerations based on ray tracing through the grating finite thickness. For the blazed grating case of Fig. 1(a), the width $w_{a}$ of the shadowing zone is simply obtained by the beam refraction at the upper grating boundary. For large period-to-wavelength ratios and for grating depths equal to $\lambda /(n-1)$, the normalized shadowing width $w_{a} / \Lambda$ is given by

$$
w_{a} / \Lambda=\frac{1}{n(n-1)}\left(\frac{\lambda}{\Lambda}\right)^{2} \text {. }
$$

For blazed-index gratings, the shadowing zone takes its origin from the nearly parabolic bending due to the propagation through a graded-index dielectric layer. The width $w_{b}$ of the shadowing zone can be derived analytically, and, for asymptotically large period-to-wavelength ratios, it is found that

$$
w_{b} / \Lambda=\frac{1}{2 n(n-1)}\left(\frac{\lambda}{\Lambda}\right)^{2},
$$

a value two times smaller than that found for $w_{a} / \Lambda$. This factor of 2 may be one intuitive explanation for the difference in diffraction efficiency observed for blazedindex and blazed gratings in Fig. 2. Of greater importance in Eqs. (1) and (2) is the dependence with $n$ of the shadowing zone; it is predicted that the use of high-index materials has a beneficial effect on the performance of the diffractive elements. Indeed, this qualitative prediction is confirmed by electromagnetic theory. Figure 3 shows the first-order diffraction efficiency of blazed-index gratings as a function of the period-to-wavelength ratio for $n$ $=1.52,2,2.5$ and for unpolarized light. Figure 3 does not clearly exemplify the net benefit of increasing the value of $n$, since, as $n$ increases, the Fresnel loss also increases. This is why the diffraction efficiency for $n$

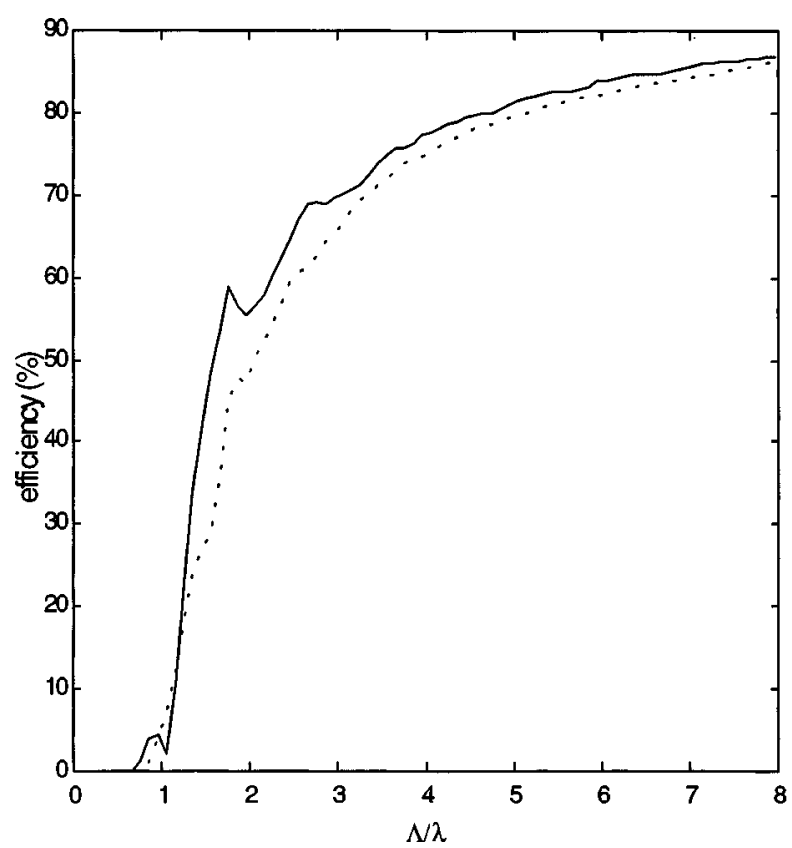

Fig. 2. First-order diffraction efficiency of the gratings considered in Fig. 1 as a function of the period-to-wavelength ratio for $n=1.52$ and for unpolarized light. Solid and dotted curves correspond to blazed-index and blazed gratings, respectively. 


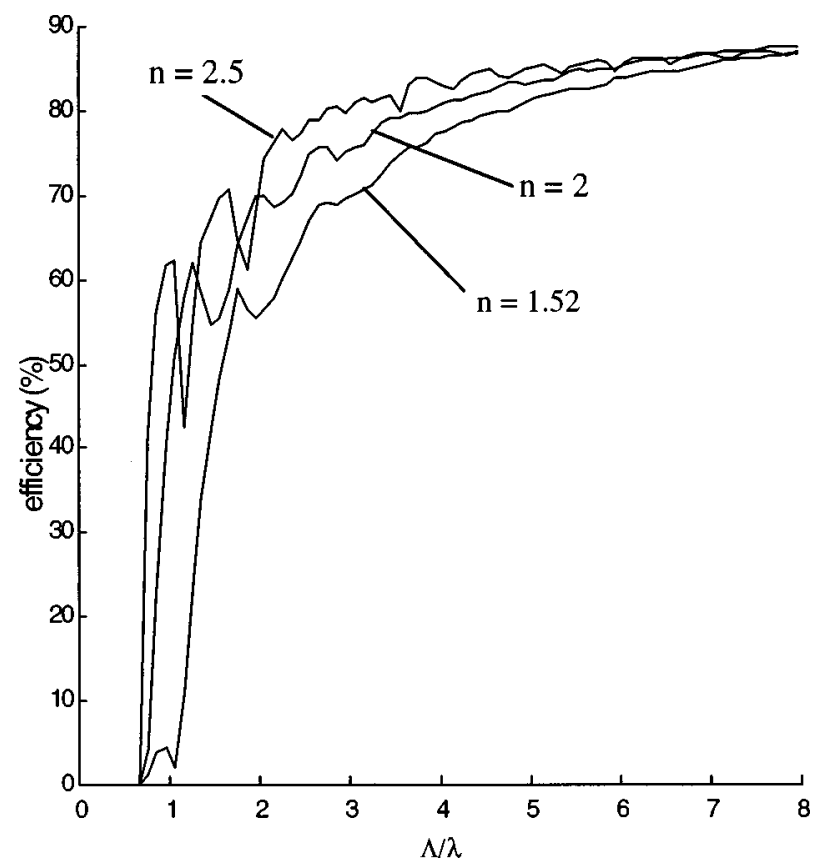

Fig. 3. First-order diffraction efficiency of blazed-index gratings as a function of the period-to-wavelength ratio for several values of $n(n=1.52,2,2.5)$ and for unpolarized light.

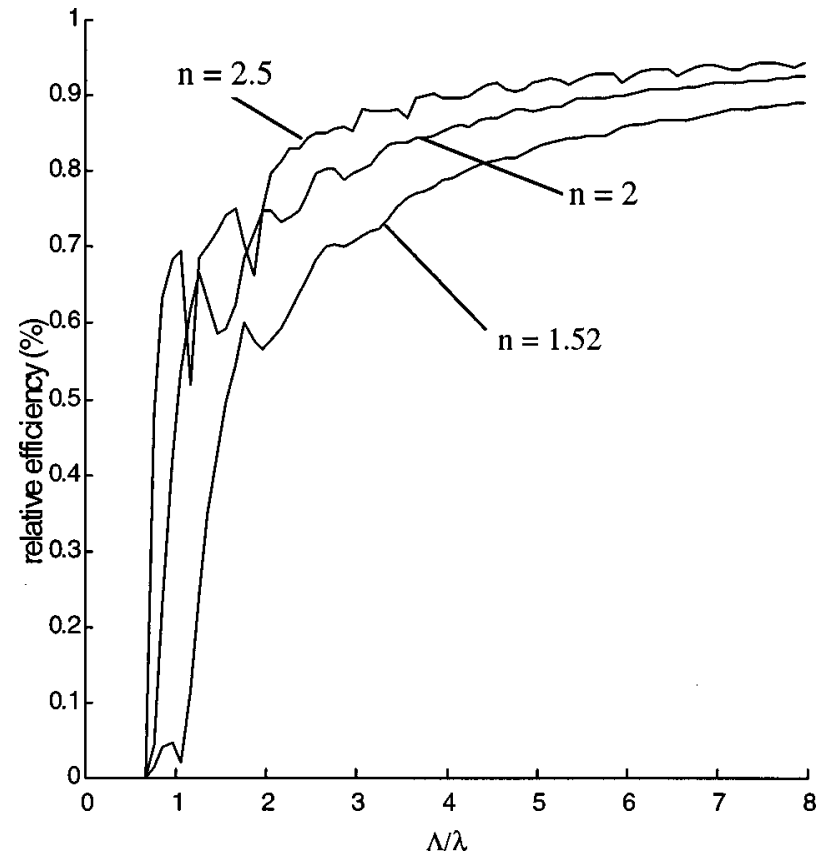

Fig. 4. Same as in Fig. 3, except that the relative efficiency, defined as the percentage of the total transmitted light diffracted into the first order, is plotted versus $\Lambda / \lambda$.

$=2.5$ becomes smaller than those obtained for $n$ $=1.52,2$ for large period-to-wavelength ratios. Figure 4 shows the relative efficiency defined as the percentage of the total transmitted light diffracted into the first order, an important figure of merit related to the effect of the spurious diffracted orders on image quality. Clearly, the use of high-index material increases this percentage and improves performance.

\section{FABRICATION OF BLAZED BINARY GRATINGS}

The fabrication of blazed-index diffractive elements through a diffusion process or ion exchange as used for fabricating graded-index lenses or waveguides is difficult because the $2 \pi$-phase jumps required at zone extremities are smoothed by the fabrication process. Conversely, artificial dielectric diffractive components composed of subwavelength features encoding continuous phase delay are easier to fabricate.

In general, the design of blazed binary diffractive components is easy. Once the phase transfer function that defines the diffractive component is known at the nominal wavelength, it is sampled at different point locations. We assume that this sampling is made on a regular $2 \mathrm{D}$ square grid. The sampling period, or, equivalently, the distance between two adjacent microstructures of the diffractive element, is denoted by $\Lambda_{1}$. Then a calibration curve that relates the phase delay for a given etch depth, or, equivalently, the effective index, to the fraction of material removed is used to associate a specific microstructure geometry to a given point location. Such a calibration curve is shown in Fig. 5, where the effective index $n_{\text {eff }}$ is plotted as a function of the fill factor of square pillars patterned in a 2.3-refractive-index material for $\Lambda_{1}$ $=272 \mathrm{~nm}$. The fill factor is defined as the ratio of the pillar width to the sampling period.

\section{A. Choice of Sampling Period and Structural Cutoff}

The sampling period chosen has to be as large as possible for ease of fabrication of the diffractive element. It is usually selected so that only one transmitted order and one reflected order are propagating in the substrate and in the incident medium (see, e.g., Refs. 12-23, 25, and 26). This choice amounts to selecting a sampling period that is smaller than the cutoff $\Lambda_{c}$, which is defined as the

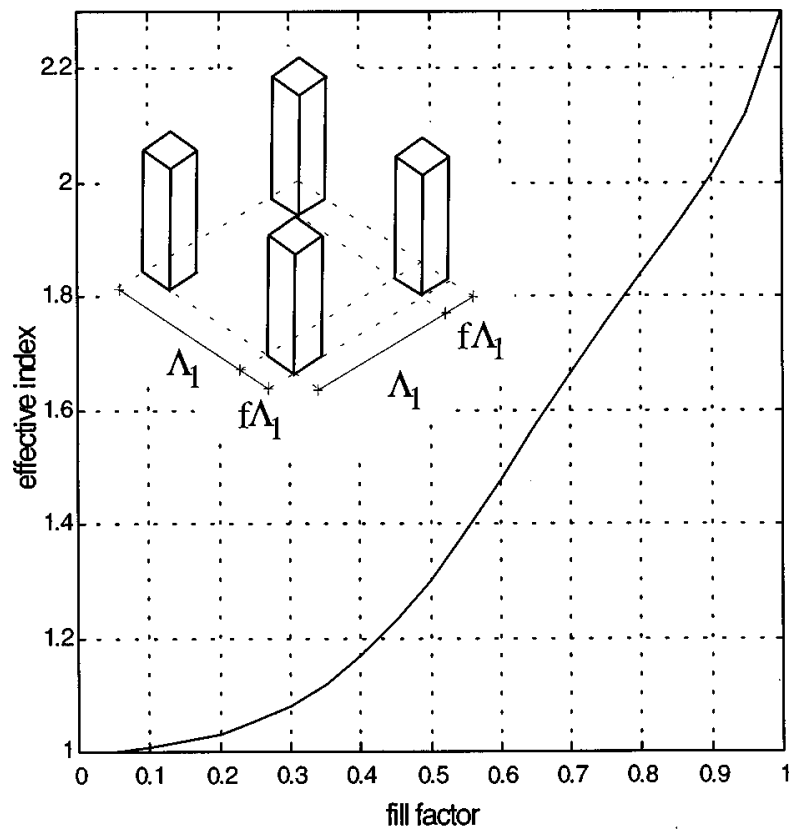

Fig. 5. Calibration curve. Effective index of a 2D grating composed of a 272-nm-period array of square pillars engraved in a 2.3-refractive-index material versus the fill factor. 
period above which nonzero diffracted orders are evanescent. In a recent study ${ }^{27}$ we quantitatively discussed how to select the sampling period and introduced a new cutoff above which the analogy between subwavelength dielectric gratings and homogeneous media ceases to be practically valid. This new cutoff $\Lambda_{s}$ was called the structural cutoff to emphasize that it is intrinsic to the grating structure. The structural cutoff of a given periodic structure is defined as the period below which only one propagating mode (this mode may be polarization dependent) is supported by the structure for any fraction of removed material. This definition relies on the assumption that, in the static limit $(\lambda \rightarrow \infty)$, 2D periodic structures support only one propagating mode. Although intuitively clear from a physical point of view, no simple demonstration of the existence and unicity of this mode is available for $2 \mathrm{D}$ gratings. Mathematically sound proofs based on homogenization theories are available, ${ }^{38}$ but understanding them requires a good background in functional analysis. A much simpler demonstration can be found in Ref. 39 for the specific case, already interesting in practice, of centrosymmetric gratings illuminated under normal incidence.

The structural cutoff value can be determined only numerically. Denoting by $x$ and $y$ the periodicity axes of the grating and by $z$ the normal to the grating boundaries, one determines the modes that are propagating inside the grating region along the $z$ direction by expanding the electromagnetic fields along the $x$ and the $y$ directions in a Fourier basis. Denoting by $\exp (j 2 \pi n z / \lambda)$ the $z$ dependence of the modes, one computes the effective index $n$ of the fundamental mode by the plane-wave method. ${ }^{33}$ The computation amounts to solving an eigenproblem. Starting from small values, one slowly increases the grating period until a second propagating mode appears. In general, this second mode appears for large pillar widths ${ }^{40}$ first, and one can reduce computational efforts by solving the eigenproblem only for large fill factor values.

When only one mode propagates in a grating (all the others are evanescent), this mode travels backward and forward between the two grating boundaries in the same way as multiple beam interference occurs in a thin film. Consequently, the zeroth-order reflected and transmitted amplitudes are approximately those of a thin film with a refractive effective index equal to the normalized wavevector modulus $n$ of this mode. ${ }^{39,41}$ Sampling with periods $\Lambda_{1}$ that are larger than the structural cutoff $\Lambda_{s}$ is problematical because the analogy between subwavelength gratings and artificial dielectrics ceases to be valid. An example of problems encountered when sampling with $\Lambda_{1}>\Lambda_{s}$ may be found in Ref. 27, where it is observed that the phase of the transmitted zeroth order exhibits a chaotic behavior and is not a monotonic function of fill factor. For another illustrative example, we consider the same synthesis problem as that of Ref. 24: The wavelength is $633 \mathrm{~nm}$, and the grating is composed of a square array of square pillars. The pillar height is $1.032 \mu \mathrm{m}$, and the sampling period is $700 \mathrm{~nm}$. This grating is etched in a quartz substrate of refractive index 1.46 and is illuminated at normal incidence from the substrate. The dotted curve in Fig. 6 represents the $n$ values of all the propagating modes. As much as five modes are propagating for large fill factors. Multiplication signs represent the zeroth-order transmitted diffraction efficiency as a function of the pillar width. ${ }^{42}$ For $f \approx 0.5$, this efficiency drops below 60\%. Conversely, the circles represent the transmitted zeroth-order efficiencies obtained for a sampling period equal to the structural cutoff $\left(\Lambda_{s}=440 \mathrm{~nm}\right)$, a value slightly larger than the cutoff $\left(\Lambda_{c}=434 \mathrm{~nm}\right)$. Efficiency values larger than $95 \%$ are obtained for any value of $f$. Following the argument of Chen and Craighead, ${ }^{24}$ namely, that large sampling periods are acceptable as long as the transmitted zeroth-order is sufficiently high, it is reasonably expected that a much better performance would have been obtained for a sampling period that was smaller than the structural cutoff.

In general, we observed that, the higher the refractive index of the material patterned, the smaller the structural cutoff. ${ }^{40}$ For example, while $\Lambda_{s}$ is as small as 272 $\mathrm{nm}$ for a 2.3-refractive-index material (see Section 4), it is much larger for glass $\left(\Lambda_{s}=440 \mathrm{~nm}\right.$ in the above example). Clearly, the larger the sampling period, the easier the fabrication. Thus one might ask whether it is judicious to consider high-refractive-index material for fabricating blazed binary diffractive components. Since this paper is specifically devoted to the synthesis and fabrication of blazed binary components in $\mathrm{TiO}_{2}$, it is crucial to realize that, while increasing the requirements on the pillar width, the use of a high-refractive-index material decreases the etching depth $h$ required for a $2 \pi$-phaseshift modulation. One relevant parameter for quantifying the fabrication difficulty is the ratio $h / \Lambda_{s}$. Assuming that a $2 \pi$-phase-shift modulation is achieved for a depth $h=\lambda /(n-1)$, with $n$ being the refractive index of the

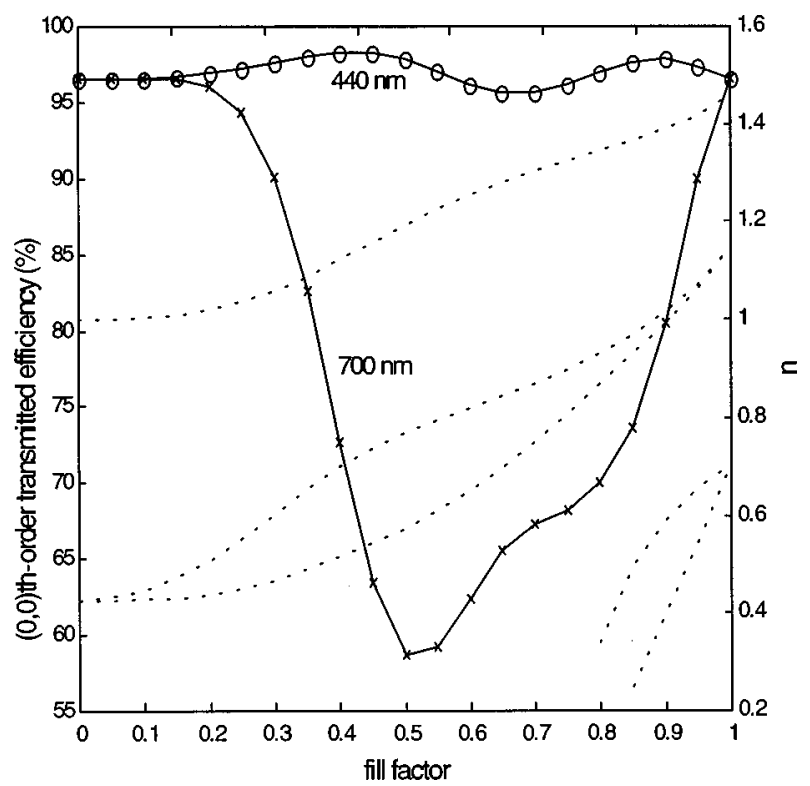

Fig. 6. Solid curves: Transmitted $(0,0)$ th-order diffraction efficiency of a $2 \mathrm{D}$ grating composed of square pillars placed on a square grid of period $\Lambda$ as a function of the fill factor. The pillar height is $1.032 \mu \mathrm{m}$, the wavelength used is $0.6328 \mu \mathrm{m}$, and the pillars are assumed to be etched in a glass substrate of refractive index 1.46. $\times$ 's, $\Lambda=700 \mathrm{~nm}$; circles, $\Lambda=440 \mathrm{~nm}$. Dotted curves: $n$ values of all the propagating modes supported by the biperiodic structure for $\Lambda=700 \mathrm{~nm}$. The upper dotted curve $(n$ varying between 1 and 1.46) corresponds to the grating effective index. 
etched material, $h / \Lambda_{s}$ is equal to 1.79 for $n=2.3$, a value that compares favorably with that of 3.13 , obtained for $n=1.46$. From this simple numerical example we can conclude that the use of a high-refractive-index material not only improves the performance of the component, as discussed in Section 2, but also relaxes fabrication constraints and decreases the aspect ratio to a point where binary blazed gratings can be fabricated with current technology.

\section{B. Fabrication}

In the experimental work of Sections 4 and 5, blazed binary diffractive components are fabricated by etching of a $\mathrm{TiO}_{2}$ layer evaporated onto a glass substrate. An $e$-beam evaporation technique with a plasma gun is used for the $\mathrm{TiO}_{2}$ coating. The plasma is composed of a 42:58 $\mathrm{Ar}-\mathrm{O}_{2}$ gas combination. The full evaporation process is optimized to obtain stable and dense layers. The deposition rate is $\approx 0.1 \mathrm{~nm} / \mathrm{s}$. After the evaporation the $\mathrm{TiO}_{2}$ layer is patterned by $e$-beam lithography and reactive ion etching (RIE). First, a poly(methyl methacrylate) (PMMA) film is spin coated on top of the $\mathrm{TiO}_{2}$ layer. Second, it is written with a JEOL JBX5D2U vector scan high-resolution pattern generator equipped with an $\mathrm{LaB}_{6}$ filament. A 50$\mathrm{keV} e$ beam resulting in a $25-\mathrm{nm}$-diameter probe beam is used during the exposure. The writing-field area of the $e$-beam generator operating in its highest resolution is $40 \mu \mathrm{m} \times 40 \mu \mathrm{m}$. After development of the PMMA, an intermediate nickel layer is $e$-beam evaporated onto the surface and is lifted off by dissolution of the PMMA. The lift-off technique improves the selectivity and the fidelity of the pattern transfer during the RIE process, which is performed in a Nextralne 110 system equipped with a silicon cathode. The etching process uses a $\mathrm{SF}_{6}(1 / 2) /$ $\mathrm{CH}_{4}(1 / 2)$ gas mixture at a pressure of $8 \mathrm{mTorr}(1.07 \mathrm{~Pa})$ with an equal flow rate for each gas and a $\mathrm{rf}$ power of 30 $\mathrm{W}$, which produces a self-induced bias voltage of $-180 \mathrm{~V}$. This process, optimized for steeper sidewalls, results in an etching rate of $\approx 40 \mathrm{~nm} / \mathrm{min}$.

\section{Fabrication Constraints}

In practice, because of limited resolution, all pillar widths are not suitable for fabrication. We denote by $\Delta_{1}$ the minimum value of the pillar width that is effectively manufacturable for a given technology. Similarly, we denote by $\Delta_{2}$ the minimum spacing between two adjacent pillars. While $\Delta_{1}$ is related to the feasibility of stable tall pillars, $\Delta_{2}$ is instead constrained by the possibility of fully etching ridges down to the substrate. We denote by $f_{1}$ and $f_{2}$ the two associated fill factors, $f_{1}=\Delta_{1} / \Lambda_{1}$ and $f_{2}$ $=1-\Delta_{2} / \Lambda_{1}$, respectively. The corresponding effective indices given by the calibration curve are denoted by $n_{\text {min }}$ and $n_{\max }$, respectively. The vertical dotted lines in Fig. 7 are the limits of the region of acceptable fill factors. In this example we chose $\Delta_{1}=\Delta_{2}=80 \mathrm{~nm}$, which was found to be compatible with our fabrication procedure. The useful interval for fill factors is [0.29; 0.71], and the effective indices $n_{\min }$ and $n_{\max }$ are equal to 1.08 and 1.66, respectively.

Because the calibration curve varies rapidly for large fill factors, the value of $n_{\max }$ is significantly smaller than 2.3. This is clearly a drawback for the fabrication, since,

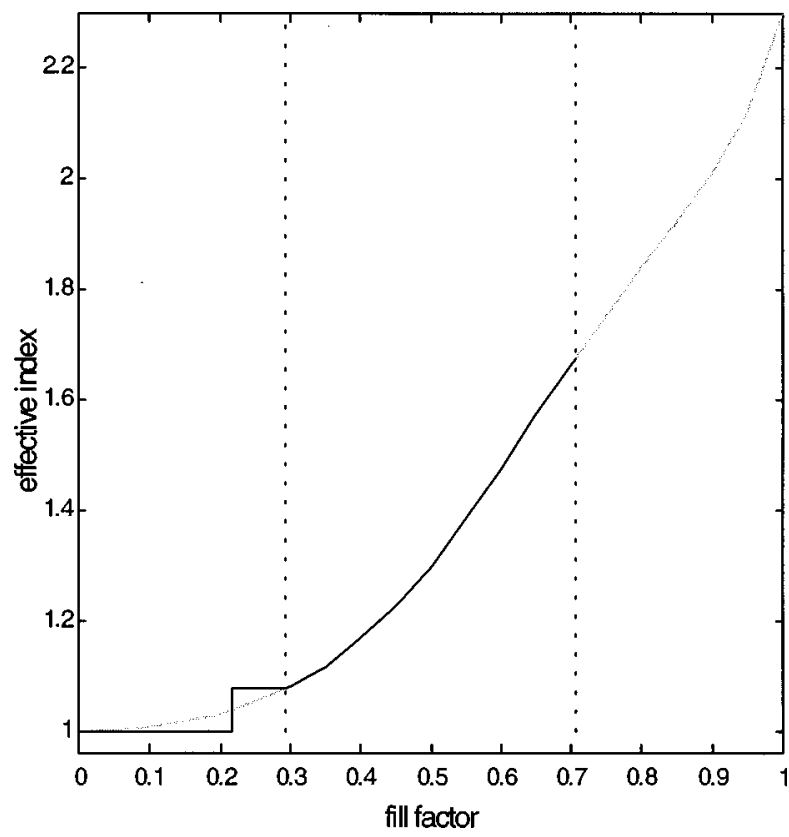

Fig. 7. Modified calibration curve. Thin curve: same as in Fig. 5. Vertical dotted lines: limits imposed by fabrication constraints for $\Delta_{1}=\Delta_{2}=80 \mathrm{~nm}$. On the left-hand side ( $f$ $<\Delta_{1} / \Lambda_{1}$ ) the pillar width is too small for stable fabrication. On the right-hand side $\left(f>1-\Delta_{2} / \Lambda_{1}\right)$, the spacing between two adjacent pillars is too narrow for a reliable RIE process. The central part $\left(\Delta_{1} / \Lambda_{1}<f<1-\Delta_{2} / \Lambda_{1}\right)$ corresponds to fill factors effectively manufacturable. Thick curve: modified calibration curve used effectively for the design of prismlike blazed binary gratings. Fill factors larger than $1-\Delta_{2} / \Lambda_{1}$ are not considered, and, for $0<f<\Delta_{1} / \Lambda_{1}$, the continuous calibration (thin) curve is replaced by a steplike function for which only two values ( 1 and $n_{\text {min }}$ ) of the effective index are encoded.

as noted in Section 1, higher performance and easier fabrication are achieved for large $n$ values. This situation can be alleviated in two ways. The first approach consists in selecting a sampling period that is slightly larger than the structural cutoff (see Section 5). The second approach consists in choosing different microstructure geometries. Following Grann et al. ${ }^{43}$ one could, for instance, consider square holes instead of square pillars. We did not investigate this issue in this study, considering that it would make the fabrication less reliable. ${ }^{44}$

Strictly speaking, only the interval $[0.29 ; 0.71]$ in Fig. 7 has to be used for the design. However, to make the fabrication easier, we extend the useful interval by considering that the absence of a pillar $(f=0)$ is a situation that is easily manufacturable. The calibration curve is modified in the following manner:

For $n_{\text {eff }}<\left(1+n_{\text {min }}\right) / 2$, we provide a full etch (no pillars) and encode $n_{\text {eff }}=1$; and

For $\left(1+n_{\text {min }}\right) / 2<n_{\text {eff }}<n_{\text {min }}$, we fabricate pillars with $f=f_{1}$ and encode $n_{\text {eff }}=n_{\text {min }}$.

The thick curve in Fig. 7 shows the effect of extending the useful interval $[0.29 ; 0.71]$ by considering the fabrication of pillars with null fill factors. All the design considerations reported hereafter are based on a similar modification of the calibration curve. Clearly, this modification introduces a systematic bias at the design stage. How- 
ever, we estimated that, while the resulting penalty in terms of diffraction efficiency never exceeds $1 \%$ or $2 \%$, the etching depth is reduced from $\lambda /\left(n_{\max }-n_{\min }\right)$ to $\lambda /\left(n_{\max }\right.$ - 1); we believe that the balance is favorable with current state-of-the-art of fabrication facilities.

\section{DESIGN OF BLAZED BINARY GRATING}

In this section we consider the design of blazed binary gratings. A component often referred to as a diffractive beam deflector or a prismlike grating, the blazed grating deserves particular attention because of its major utility in various fields, including micro-optics and spectrometry.

\section{A. Design}

As above, a 2D square grid is assumed for sampling. We denote by $x$ and $y$ the main axes of this grid. The grating period along the $x$ direction is denoted by $\Lambda$. For a given application and a given deviation angle, the period is straightforwardly obtained by use of the grating equation. We first divide the period into $N$ intervals. The interval length corresponds to the sampling period $\Lambda_{1}, \Lambda_{1}$ $=\Lambda / N$. Because a locally periodic square grid with square pillars yields the best approximation to an isotropic effective index, at least under normal incidence, the grating period along the $y$ direction is also $\Lambda_{1}$. We then choose the integer $N$ such that $\Lambda / N \leqslant \Lambda_{s}$ and $\Lambda /(N$ - 1) $>\Lambda_{s}$, i.e., just less than or equal to the structural cutoff. At the center of each interval (sampling points marked by x's in Fig. 8), we associate an effective index value $n(i), \quad i=1,2, \ldots N$, so that $n(1)=1, n(N)$ $=n_{\max }$, and $n$ is linearly varying from 1 to $n_{\max }$ :

$$
n(i)=\frac{n_{\max }-1}{N-1}(i-1)+1 .
$$

For a selected microstructure geometry, the effective index is then computed as a function of the microstructure width. Including fabrication constraints (parameters $\Delta_{1}$ and $\Delta_{2}$ for pillar microstructures, for instance), a modified calibration curve is obtained as in Fig. 7. From this curve the set of $N$ microstructure widths is derived. The grating geometry is now defined, and only the grating depth has to be fixed to complete the design. We simply set the grating depth, using scalar theory. Noting that the index modulation $\left(n_{\max }-1\right)$ is achieved on $(N-1)$

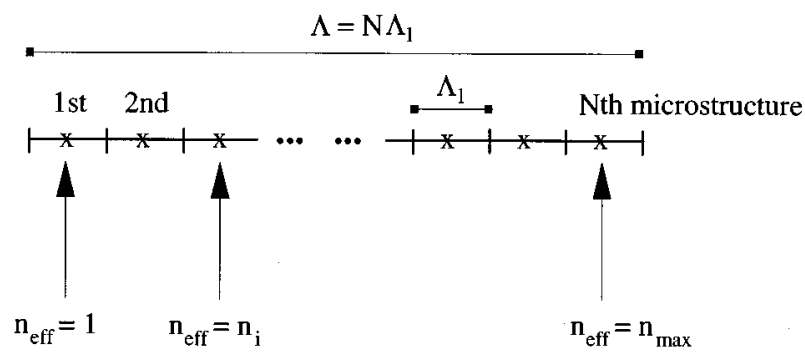

Fig. 8. Design procedure 1 for prismlike blazed binary gratings. One period of length $\Lambda$ is shown. This period is divided into $N$ intervals. The sampling period is equal to $\Lambda_{1}=\Lambda / N$. The x's at the interval centers indicate the location of the sampling points. The effective indices associated with every sampling point are denoted by $n(i), i=1,2, \ldots N$ and are linearly varying between 1 and $n_{\max }$ according to Eq. (3). sampling periods (see Fig. 8) and hence that the total index modulation is $N /(N-1)\left(n_{\max }-1\right)$, we fix the grating depth $h$ by $^{45}$

$$
h=\frac{N-1}{N} \frac{\lambda}{n_{\max }-1} .
$$

Hereafter this design procedure will be referred to as procedure 1. It is straightforward and does not rely on any iterative technique.

Because efficient electromagnetic theories are now available for computing the diffraction efficiencies of $2 \mathrm{D}$ gratings, refinements on the transition-point locations and grating depth are feasible but extremely demanding in terms of computation time. Subsection 4.C illustrates this opportunity. However, from several tests whose details are not reported in this paper, we found that the gratings designed through procedure 1 provide high diffraction efficiencies (even for period-to-wavelength ratios as small as 1.5) and that refinements with electromagnetic theory do not provide substantial improvements.

The choice of setting the effective indices 1 and $n_{\max }$ to the first and the last sampling points, respectively, as shown in Fig. 8, requires a few comments. A more natural choice, inspired from the standard blazed grating, consists in setting the effective index values 1 and $n_{\text {max }}$ to the two period extremities. The corresponding grating depth would be $\lambda /\left(n_{\max }-1\right)$, which coincides with Eq. (4) for large $N$ values. In fact, as mentioned above, procedure 1 artificially increases the index modulation by extending the interval $\left[1 ; n_{\text {max }}\right.$ ] to effective indices smaller than 1 on one side and effective indices larger than $n_{\max }$ on the other side. The net benefit is a reduction of fabrication complexity. To illustrate our purpose, let us consider a $3 \lambda$-period blazed binary subwavelength grating etched in $\mathrm{TiO}_{2}$ for $\mathrm{He}-\mathrm{Ne}$ operation. Assuming the modified calibration curve of Fig. 7 and $\Delta_{2}=80 \mathrm{~nm}$, the maximal effective index $n_{\text {max }}$ is 1.664 . Since the $3 \lambda$ period is exactly divided into $N=7$ intervals for a sampling period of 272 $\mathrm{nm}$, the grating depth is equal to $817 \mathrm{~nm}$, according to Eq. (4). If we now set the effective index values 1 and $n_{\text {max }}$ to the two period extremities, the corresponding grating depth $\lambda /\left(n_{\max }-1\right)$ is equal to $953 \mathrm{~nm}$, a value $130 \mathrm{~nm}$ larger than that obtained with procedure 1 . This depth reduction is significant from the fabrication point of view and can be even more important for smaller $N$ values. We conclude that procedure 1 is especially relevant for the design of blazed binary gratings with small periods. At large periods, $N$ is large, and the grating depth is simply given by $\lambda /\left(n_{\max }-1\right)$.

\section{B. Theoretical Performance}

We now proceed with a quantitative analysis of the performance achieved by gratings designed through procedure 1 with the modified calibration curve of Fig. 7 . This situation corresponds to a blazed binary grating etched in $\mathrm{TiO}_{2}$ for operation at $633 \mathrm{~nm}$ with a sampling period equal to the structural cutoff, $272 \mathrm{~nm}$. The procedure is tested for several values of the integer $N$ and thus for different period-to-wavelength ratios. Figure 9 shows the first-order diffraction efficiency as a function of the 


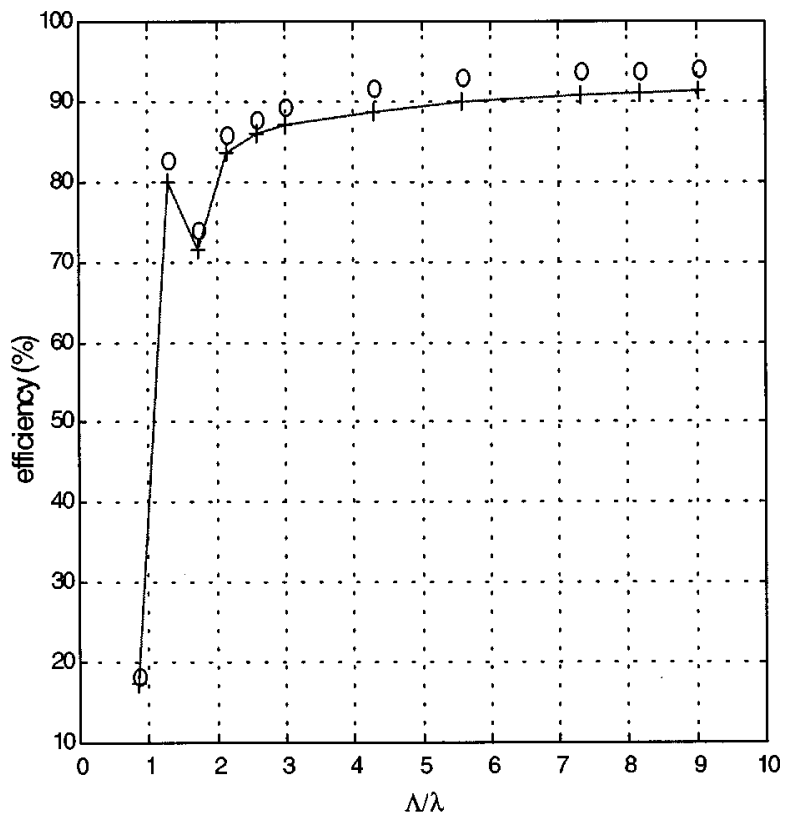

Fig. 9. Theoretical performance for design procedure 1 . The diffraction efficiency of blazed binary gratings with a 272-nm sampling period is considered for different period-to-wavelength ratios. Both unpolarized light and normal incidence from air are assumed for the computation. Solid curve: first-order diffraction efficiencies; circles: percentage of the total transmitted light diffracted into the first order.

period-to-wavelength ratio. The circles correspond to the percentage of the total transmitted light diffracted into the first order.

We conducted substantial tests for checking the validity of the numerical results by increasing the number of retained orders. For instance, well-converged results were obtained for $N=10$ (points at abscissa $\Lambda / \lambda=4.3$ in Fig. 9) with 9 and 51 retained orders in the $y$ and the $x$ directions, respectively. For larger periods, we increased the number of retained orders in the $x$ direction, with this number reaching 67 for $N=21(\Lambda / \lambda=9.02)$.

\section{Experimental Results}

We now apply the theoretical analysis of Subsections 4.A and 4.B to the design, fabrication, and testing of a $3 \lambda$ period blazed binary subwavelength grating etched in $\mathrm{TiO}_{2}$ for operation with unpolarized light at $633 \mathrm{~nm}$. The $3 \lambda$ period corresponds to nearly $20^{\circ}$ deflection into air. For a 272-nm sampling period, the grating period along the $x$ axis is exactly divided into seven sampling periods. Our design strictly follows procedure 1 , and we determine the seven pillar widths according to the modified calibration curve of Fig. 7. Then, assuming unpolarized light and normal incidence from air, we vary the grating depth and maximize the diffraction efficiency of the first order. For a $817-\mathrm{nm}$ depth, we obtained $87 \%$. The value of 817 $\mathrm{nm}$ is very close to the scalar prediction $(6 / 7)\left[\lambda /\left(n_{\max }\right.\right.$ $-1)] \approx 816 \mathrm{~nm}$ of procedure 1 . In a second step, we optimize the pillar-center locations along the blazed profile direction, preserving their square shapes and fill factors. The optimization was performed with a gradient-descent algorithm starting with small random perturbations for the free parameters. Only a few modifications were ob- tained: The narrowest pillar was appreciably shifted toward the region of small effective index values. We end up with a diffraction efficiency of $88 \%$. The diffraction values of the other transmitted diffraction orders are given in parentheses in Table 1 . The percentage of the total transmitted light diffracted into the first order is $91 \%$. For a wave normally incident from the glass substrate, rather similar numerical predictions were obtained: We found that the first-order diffraction efficiency is also $88 \%$ and that the percentage of the total transmitted light diffracted into the first order is $93 \%$.

We indeed admit that our optimization procedure is suboptimal. Searching for a global optimal in terms of diffraction efficiency would, however, result in a rather lengthy procedure that is quite impracticable with today's computers because the analysis of $2 \mathrm{D}$ gratings is computationally expensive. Nevertheless, we believe that procedure 1 provides nearly optimal solutions and that an extensive search for refinements with electromagnetic theory will not drastically improve the performance.

The grating fabrication involves $e$-beam writing in a 150-nm-thick PMMA-layer and fluorine lift-off with a 30nm-thick nickel mask. The RIE step lasts $23 \mathrm{~min}$. The grating pattern is written over a $204 \mu \mathrm{m} \times 228 \mu \mathrm{m}$ area. The $e$-beam process lasts 4 min. A scanning-electron photograph of the grating is shown in Fig. 10. On the vertical and horizontal axes, periods are $\Lambda=1.9 \mu \mathrm{m}$ and $\Lambda_{1}$, respectively. The grating was tested with a $\mathrm{He}-\mathrm{Ne}$ laser at normal incidence from air. The laser beam waist is focused with a lens of 45-mm focal length. We estimate that more than $99 \%$ of the incoming light passes through the grating aperture. We determine the diffraction efficiencies by measuring the powers of the diffracted beams and dividing them by the power of the incident beam. Measurements have been corrected for Fresnel losses incurred at the back side of the glass substrate. Table 1 shows the measured efficiencies of the different transmitted orders for TE and TM polarizations. Their corresponding values, computed with electromagnetic theory, are given in parentheses. Deviations between numerical prediction and measurement are noticeable, especially for TE polarization. We also observed that the first-order diffraction efficiency is weakly dependent on polarization: We found $80 \%$ and $84 \%$ for TE and TM polarizations, respectively-values approximately $7 \%$ lower than those predicted by electromagnetic theory. The highest efficiency $(\approx 3.5 \%)$ of the five transmitted orders (except the first one) is observed for TE polarization and for the second order. Although deviations between experiments and theory are significant, the experimental results are good.

As a matter of comparison, it is noteworthy that the maximum diffraction efficiency ${ }^{46}$ achieved by a blazed échelette grating in glass with a $3 \lambda$ period is $66.5 \%$, a theoretical value $15 \%$ smaller than that obtained experimentally in this study. Moreover, it is interesting to compare the experimental and the theoretical results obtained in this study with those reported in Ref. 27 by the same authors. They can be directly compared, since they are both relative to the same diffractive element, a prismlike grating with a $3 \lambda$ period etched into $\mathrm{TiO}_{2}$. The only difference between these two works is that a sampling pe- 
Table 1. Diffraction Efficiencies (in Percent) of the Different Transmitted Orders for the Grating Shown in Fig. 10 ${ }^{a}$

\begin{tabular}{|c|c|c|c|c|c|c|c|c|c|}
\hline Order & 4 & 3 & 2 & 1 & 0 & -1 & -2 & -3 & -4 \\
\hline \multicolumn{10}{|l|}{$\mathrm{TE}$} \\
\hline & $\begin{array}{c}- \\
(0.4)\end{array}$ & $\begin{array}{c}- \\
(0.2)\end{array}$ & $\begin{array}{c}3.5 \\
(1.3)\end{array}$ & $\begin{array}{l}80 \\
(87.8)\end{array}$ & $\begin{array}{c}2.5 \\
(1.6)\end{array}$ & $\begin{array}{l}2 \\
(1.7)\end{array}$ & $\begin{array}{c}2.5 \\
(2.9)\end{array}$ & $\begin{array}{c}- \\
(0.8)\end{array}$ & $\begin{array}{c}- \\
(0.3)\end{array}$ \\
\hline TM & $\begin{array}{c}- \\
(1.3)\end{array}$ & $\begin{array}{c}- \\
(0.8)\end{array}$ & $\begin{array}{l}2 \\
(0.7)\end{array}$ & $\begin{array}{l}84 \\
(88.8)\end{array}$ & $\begin{array}{c}1.5 \\
(1.2)\end{array}$ & $\begin{array}{c}1 \\
(1.1)\end{array}$ & $\begin{array}{l}2 \\
(1.6)\end{array}$ & $\begin{array}{c}- \\
(1.8)\end{array}$ & $\begin{array}{c}- \\
(0.1)\end{array}$ \\
\hline
\end{tabular}

${ }^{a}$ TE and TM polarizations correspond to normally incident waves from air polarized perpendicular and parallel to the $x$ and the $y$ directions, respectively. Theoretical results are given in parentheses. One obtains them by retaining 7 orders along the $y$ axis and 25 orders along the $x$ axis. The diffraction efficiency values of orders $-4,4,-3$, and 3 are not indicated because these orders are totally reflected in the substrate.

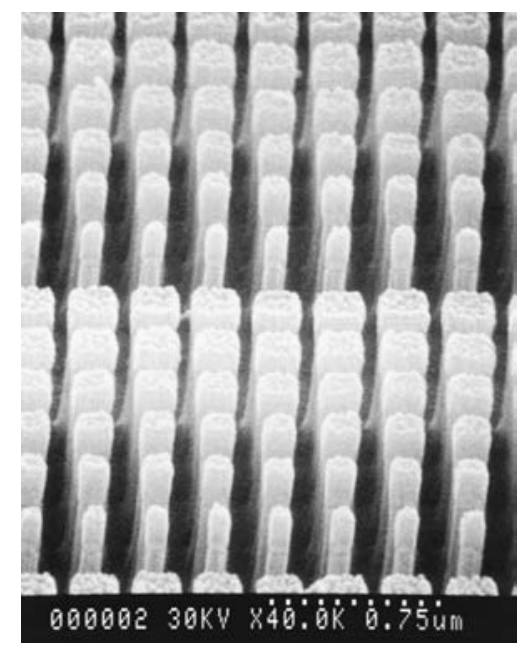

Fig. 10. Scanning-electron micrograph of a blazed binary subwavelength grating etched in $\mathrm{TiO}_{2}$. The period along the vertical axis is $1.9 \mu \mathrm{m}$, and the period along the horizontal axis is equal to the sampling period $(272 \mathrm{~nm})$. The grating depth is $\approx 816 \mathrm{~nm}$, and the maximum pillar aspect ratio is $\approx 8.8$.

riod of $380 \mathrm{~nm}$, significantly larger than the structural cutoff, was considered in Ref. 28 , where efficiencies of $82 \%$ and $77.5 \%$ were theoretically and experimentally obtained, two values that are $6 \%$ and $5 \%$ smaller, respectively, than those obtained in the present study. ${ }^{47} \mathrm{We}$ can conclude that the use of a sampling period equal to the structural cutoff is globally fruitful.

\section{BLAZED BINARY KINOFORMS}

Procedure 1, used to design prismlike gratings in Section 4 , has to be slightly modified for synthesizing arbitrary diffractive components. The reason is that the grating depth, as defined by Eq. (4), depends on the number of sampling intervals per period and therefore on the grating period itself. Considering, for example, the design of diffractive lenses, Fresnel zones of different heights have to be considered, which is clearly unrealistic from the fabrication point of view. In this section we consider a slightly different procedure that is valid for synthesizing arbitrary diffractive phase elements. Of course, it can also be applied to the design of prismlike gratings.

\section{A. Design}

In general, a diffractive component is defined by a phase transfer function $\phi(x, y)$ for a nominal wavelength $\lambda$, $\phi(x, y) \in[0 ; 2 \pi]$. Assuming that the sampling points $(i, j)$ are located on a regular $2 \mathrm{D}$ square grid, a set of phase values $\phi_{i j}$ is defined, with $i$ and $j$ being integers. For a given calibration curve, the value of $n_{\max }$ is given. Denoting by $\phi_{M}$ the maximum value of the $\phi_{i j}$ values, $\phi_{M}<2 \pi$, we fix the thickness $h$ of the diffractive element by using

$$
h=\frac{\phi_{M}}{2 \pi} \frac{\lambda}{n_{\max }-1} .
$$

When the phase modulation is $2 \pi$, as is generally the case, the thickness $h$ is simply equal to $\lambda /\left(n_{\max }-1\right)$. Then we associate with each phase value $\phi_{i j}$ an effective index $n_{i j}$, given by ${ }^{48}$

$$
n_{i j}=\left(n_{\max }-1\right) \frac{\phi_{i j}}{\phi_{M}}+1 .
$$

From the modified calibration curve, the microstructures are deduced at every sampling point. This design procedure, referred to as procedure 2 hereafter, is simple and does not rely on any iterative techniques.

\section{B. Theoretical Performance}

We now apply procedure 2 to the design of diffractive components for operation with vertical-cavity surfaceemitting lasers at $860 \mathrm{~nm}$. Again, we consider pillars etched in $\mathrm{TiO}_{2}$ (refractive index of 2.23 at this wavelength). The sampling period $\Lambda_{1}$ is chosen as follows. For a given value of $\Delta_{2}, \Lambda_{1}$ is selected such that, for $f$ $<\left(\Lambda_{1}-\Delta_{2}\right) / \Lambda_{1}$, only one propagating mode is supported by the microstructures and, for $f>\left(\Lambda_{1}\right.$ $\left.-\Delta_{2}\right) / \Lambda_{1}$, at least two modes propagate. In this way, we can consider sampling periods slightly larger than the structural cutoff as relaxing fabrication constraints while still preserving the full analogy with homogeneous artificial dielectrics. An example is given in Fig. 11 for $\Delta_{2}$ $=75 \mathrm{~nm}$. The thin curves represent the effective indices $n$ of the propagating modes. The sampling period $\left(\Lambda_{1}\right.$ $=405 \mathrm{~nm})$ is $10 \%$ larger than the structural cutoff $\left(\Lambda_{s}\right.$ $=370 \mathrm{~nm})$ but remains much smaller than the cutoff $\left(\Lambda_{c}=566 \mathrm{~nm}\right)$.

To assess the performance of diffractive elements based on design procedure 2, we first consider prismlike gratings. Figure 12 shows the first-order diffraction efficiency as a function of the normalized depth for different periods. The gratings are designed according to procedure 2. The normalized depth is defined by the ratio be- 
tween the actual depth and the value given by Eq. (5). Thus a grating with a normalized depth equal to unity is strictly designed along the lines of procedure 2 . Two interesting features appear in Fig. 12. First, it is noticeable that the maximum efficiency is achieved for a thickness equal to or very close to $\lambda /\left(n_{\max }-1\right)$ and that this efficiency is rather large. This result is expected for large period-to-wavelength ratios where the scalar theory holds, but it is more surprising for shorter periods in the resonance domain. Applying the numerical prediction of Fig. 12 to the local periodicity of a diffractive lens, we find that the outer zone of an f/0.5 lens has a diffraction efficiency of $82 \%$. Another interesting feature is the fact that the predicted diffraction efficiencies in Fig. 12 are significantly higher than those obtained for the blazedindex gratings shown in Fig. 3. For example, for a $4.2 \lambda$ period, the efficiency is $88.5 \%$ for $n_{\max } \approx 1.86$, whereas it is only $79 \%$ and $82 \%$ in Fig. 3 for $n=1.52$ and $n=2$, respectively. Similar results are also observed for blazed binary gratings designed along the lines of procedure 1 (see Fig. 9).

\section{Sampling Effect}

The fact that blazed binary gratings designed according to procedures 1 and 2 offer diffraction efficiencies significantly larger than those achieved by the corresponding blazed-index gratings is questionable. This effect, herein called the sampling effect, was not expected by us before we conducted this study, and during the course of the study it made us doubt for a while the accuracy of our numerical predictions.

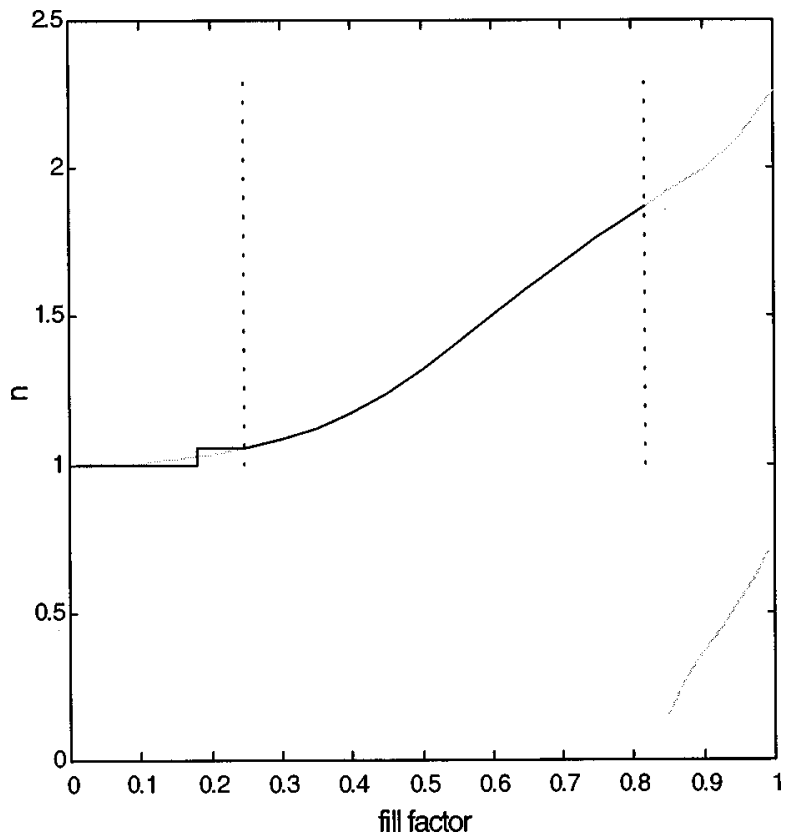

Fig. 11. Calibration curve used for the design of blazed binary diffractive components as a function of the fill factors. Thin curves: $n$ values of all the propagating modes supported by a biperiodic structure composed of a 405-nm-period array of square pillars engraved in a 2.23-refractive-index material. The upper curve ( $n$ varying from 1 to 2.23 ) corresponds to the fundamental mode or effective index. Vertical dotted lines: limits imposed by fabrication constraints for $\Delta_{1}=100 \mathrm{~nm}$ and $\Delta_{2}=75 \mathrm{~nm}$. Thick curve: modified calibration curve.

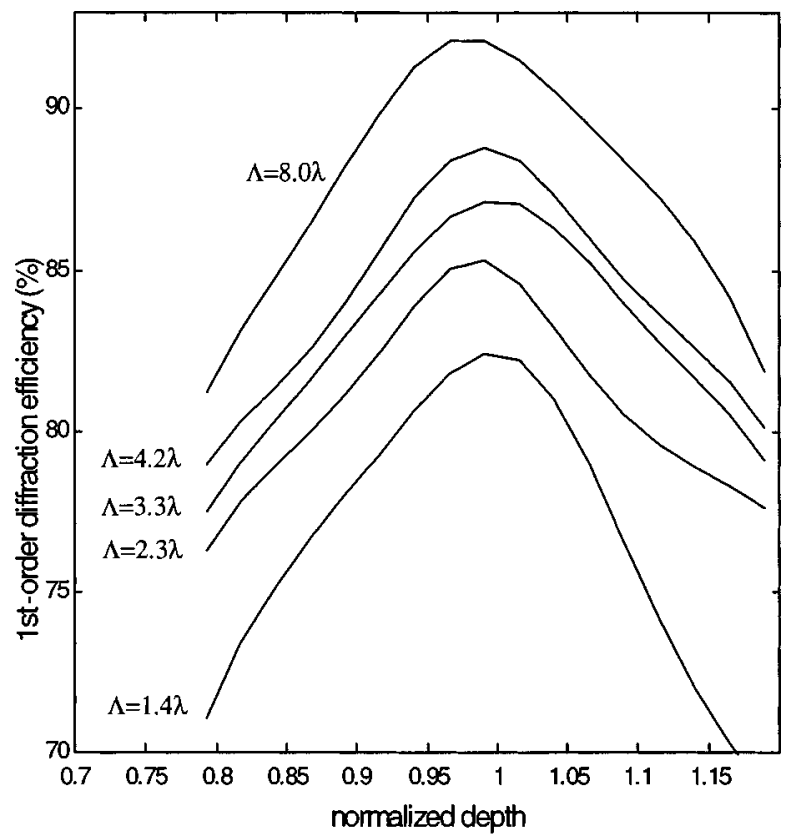

Fig. 12. Theoretical performance for design procedure 2. Firstorder diffraction efficiency of blazed binary subwavelength gratings as a function of the normalized depth for different grating periods $\Lambda \approx 1.4 \lambda, 2.3 \lambda, 3.3 \lambda, 4.2 \lambda, 8.0 \lambda$. The numerical values are obtained for $\lambda=860 \mathrm{~nm}$, for gratings etched in a $\mathrm{TiO}_{2}$ layer (refractive index, 2.23), for normal incidence from air, and for a 405-nm sampling period.

To verify this unexpected effect, we apply procedure 2 to the design of two prismlike gratings whose periods are $4.23 \lambda$ and $1.41 \lambda$. For each grating, we consider several sampling periods, and, for each sampling period, a new calibration curve relating the effective index to the fill factor is computed. In this theoretical study we allow for infinitely small pillars to be fabricated $\left(\Delta_{1}=0\right)$, and $\Delta_{2}$ is chosen such that the higher-effective-index value $n_{\max }$ is equal to 1.86. The numerical results are shown as circles in Fig. 13. For example, the values obtained for $\Lambda$ $=4.23 \lambda$ were computed for $N=9,10,11,12,13,17,19$, 21. The horizontal dotted lines represent the diffraction efficiencies of the two associated blazed-index gratings $[n=1.86$ and $h=\lambda /(n-1)]$. As predicted above, we find that the use of large sampling periods has a beneficial effect on the diffraction efficiency. This is especially true for the $1.41 \lambda$-period case, in which the diffraction efficiency for $\Lambda_{1}=400 \mathrm{~nm}$ is $20 \%$ larger than the efficiency for $\Lambda_{1}=50 \mathrm{~nm}$. Moreover, it is interesting that, for instance, in Fig. 13, the optimal sampling period is not equal to the structural cutoff of $405 \mathrm{~nm}$. For $\Lambda=1.41 \lambda$ it could be larger, and for $\Lambda=4.23 \lambda$ it could be smaller (an optimal value is found for $\Lambda_{1} \approx 370 \mathrm{~nm}$ ).

\section{Experimental Results}

We consider as experimental evidence the fabrication of an off-axis diffractive lens for operation at $860 \mathrm{~nm}$. The focal length is $400 \mu \mathrm{m}$, the off-axis angle is $20^{\circ}$, and the lens aperture is square, with a side equal to $200 \mu \mathrm{m}$. For these values, the minimum and the maximum zone widths are $1.91 \lambda$ and $8.83 \lambda$, respectively. Half the lens area consists of zones with a width smaller than $2.8 \lambda$. The lens design is performed by use of procedure 2 with 
the modified calibration curve of Fig. 11. The corresponding aspect ratio for the thinner pillars is approximately 10 . The fabrication process is the same as that described in Subsection 3.B. After evaporation of a 990nm-thick $\mathrm{TiO}_{2}$ layer onto a glass substrate, the fabrication involves $e$-beam writing in a 150 -nm-thick PMMAlayer lift-off with a 40-nm-thick nickel mask and RIE. The $e$-beam writing process over the $200 \mu \mathrm{m} \times 200 \mu \mathrm{m}$ area lasts $5 \mathrm{~min}$. Feature-size data and $e$-beam dose are adjusted simultaneously to yield pillar sizes as close as possible to those desired. Three different doses are accordingly used for narrow, intermediate, and large pillars. The fluorine etching step lasts $25 \mathrm{~min}$. A scanningelectron micrograph is shown in Fig. 14. According to the numerical results of Fig. 12, an $85.5 \%$ diffraction efficiency is expected for this lens.

The lens was tested with a vertical-cavity surfaceemitting laser emitting a Gaussian beam circularly polarized at $860 \mathrm{~nm}$. The beam waist is $1.25 \mu \mathrm{m}$. For the following tests, the laser is centered relative to the lens aperture (this corresponds to a $20^{\circ}$ angle deviation for the diffracted beam), and it is positioned in the front focal plane. We estimate that more than $99 \%$ of the incoming light passes through the lens aperture. The efficiency performance of the lens is characterized by a measurement of the first-order diffraction efficiency. We obtain an efficiency of $80 \%$ (a value $5 \%$ lower than the theoretical prediction) by measuring the power of the diffracted beam, dividing it by the power of the incident beam, and correcting for Fresnel losses incurred at the back side of the glass substrate. In addition to efficiency, spot quality

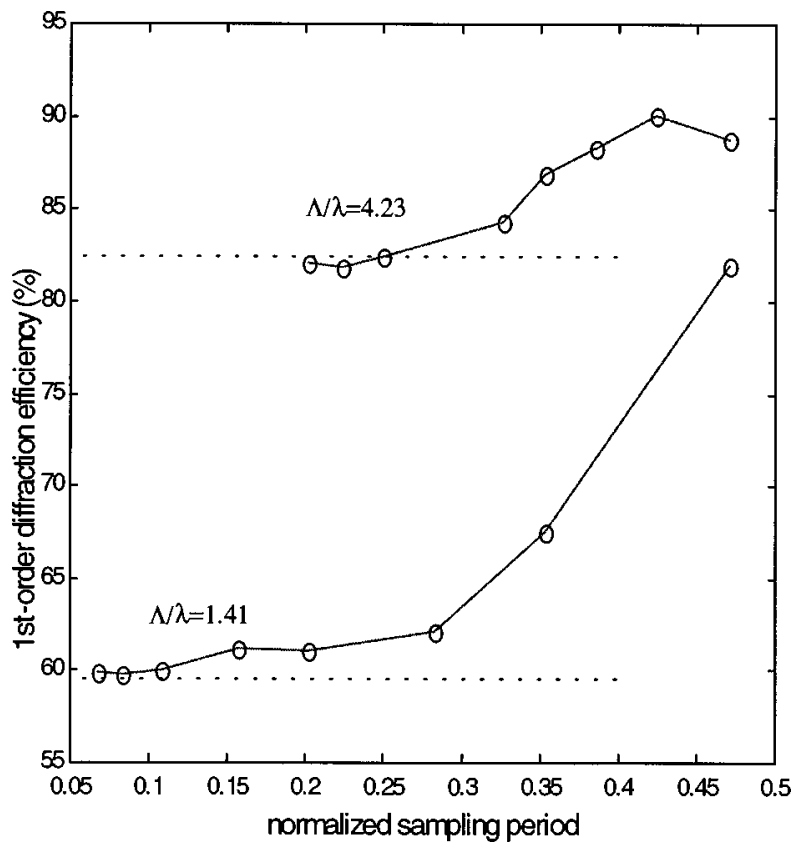

Fig. 13. First-order diffraction efficiency of blazed binary subwavelength gratings as a function of $\Lambda_{1} / \lambda$ for $\Lambda=1.41 \lambda, 4.23 \lambda$. The numerical values are obtained for $\lambda=860 \mathrm{~nm}$ and for gratings etched in $\mathrm{a}^{-} \mathrm{Ti}_{2}$ material (refractive index, 2.23). The gratings are designed according to procedure 2 with $\Delta_{1}=0$. For each sampling period, $\Delta_{2}$ is chosen so that $n_{\max }$ is equal to 1.87 . The two horizontal dotted lines correspond to the first-order diffraction efficiencies of two blazed-index gratings for $n=1.87$ and for $\Lambda=1.41 \lambda, 4.23 \lambda$.

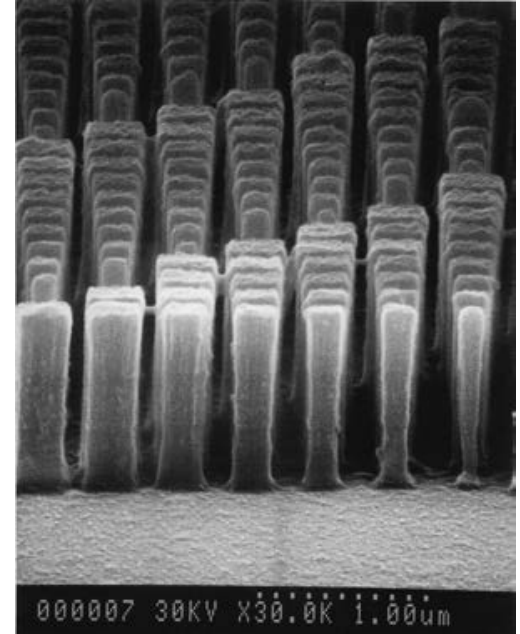

Fig. 14. Scanning-electron micrograph (located not far from a corner) of the off-axis diffractive lens.

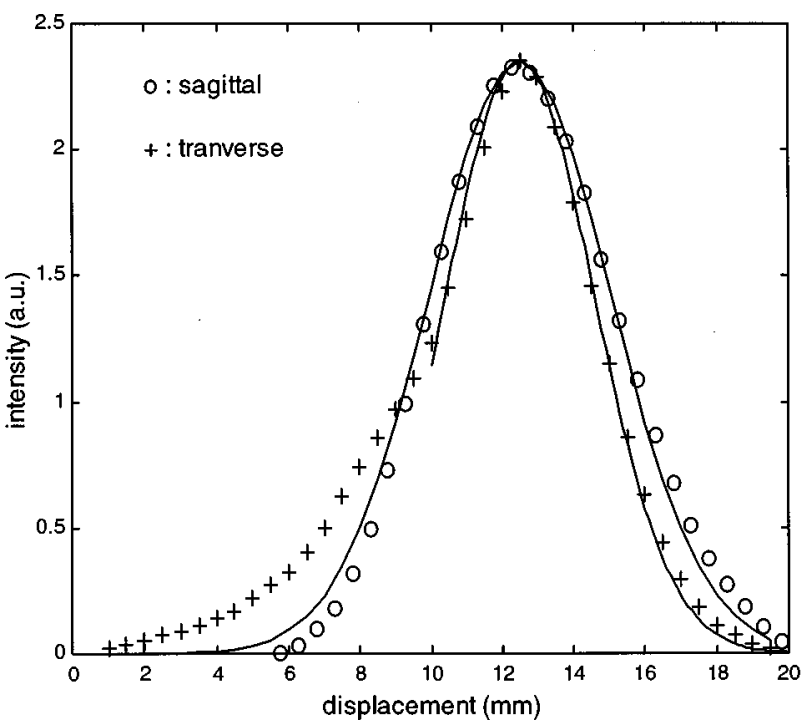

Fig. 15. Spot profiles measured at a rear distance of $400 \mathrm{~mm}$ with a $400-\mu \mathrm{m}$-diameter photodiode. Solid curves are fits by Gaussian functions.

is an important measure of lens performance. Figure 15 represents the point-spread function measured at a rear distance of $400 \mathrm{~mm}$ with a $400-\mu \mathrm{m}$-diameter photodiode. The data denoted by circles are obtained in the sagittal plane, and those denoted by plus signs are obtained in the transverse plane, perpendicularly to the off-axis direction. The long tail obtained for small displacements and for the transverse case is due mainly to coma and astigmatism because the lens was designed for on-axis operation. The two solid curves correspond to fits by Gaussian functions whose $1 / e^{2}$ contours are equal to 6 and $7.2 \mathrm{~mm}$ in diameter, respectively. These values agree rather well with the theoretical value of $7 \mathrm{~mm}$. Further analysis will be pursued to accurately quantify the lens behavior.

\section{CONCLUSIONS}

The design and the fabrication of polarization-insensitive blazed binary diffractive components for visible-light op- 
eration have been examined. Two straightforward and complementary procedures for designing such components have been proposed and quantitatively tested with electromagnetic theory. These procedures exploit the strong analogy between two-dimensional gratings with periods smaller than the structural cutoff and homogeneous thin films, take into account fabrication constraints, and provide highly efficient designs. Numerical results show that blazed binary diffractive elements etched in a high-index film consistently outperform standard blazed components in glass. The procedures exposed in this study are attractive because they provide simple, noniterative, nearly optimal, and polarization-independent ${ }^{49}$ designs even for gratings with small period-to-wavelength ratios or for high-speed lenses. Perhaps additional methods based on electromagnetic theory can be used to further enhance the performance of the blazed binary components by a local refinement $t^{50,51}$ of the subwavelength microstructure locations.

In addition, the feasibility of highly efficient diffractive components with sampling periods equal to the structural cutoff has been demonstrated. These components include a $3 \lambda$-period prismlike grating with an $82 \%$ experimental efficiency for operation at $633 \mathrm{~nm}$ and a $20^{\circ}$ offaxis diffractive lens with an $80 \%$ experimental efficiency for operation at $860 \mathrm{~nm}$. The fabrication relies on lithography and etching processes developed for the semiconductor industry and involves electron-beam writing in a PMMA layer, lift-off with a nickel mask, and reactive ion etching in a $\mathrm{TiO}_{2}$ film evaporated onto a glass substrate.

Why do blazed binary diffractive components with sampling periods approximately equal to the structural cutoff significantly outperform conventional blazed échelette gratings? We have not yet fully answered this question, but a first insight has been provided. First (with reference to Fig. 1), we pointed out that, for a given material or, equivalently, for a given refractive index, the shadowing zone of blazed échelette elements is twice as big as that of the corresponding blazed-index elements. These predictions, based on simple and approximate considerations, were confirmed by numerical results obtained with electromagnetic theory. This simple consideration does not fully explain the difference, in terms of efficiency, observed by numerical computation. Second, we provided numerical evidence that the performance of blazed binary gratings strongly depends on the sampling period. We observed that large efficiencies are obtained for large sampling periods approximately equal to the structural cutoff. In other words, efficiencies obtained with blazed binary diffractive elements designed in the static limit, $\Lambda_{1} \rightarrow 0$ (this would be a natural choice for one ideally equipped with a technology offering a resolution much smaller than the wavelength), are lower than those achieved with finite sampling periods, at least in the resonance domain. We are currently investigating some physical reasons for this result.

We can conclude from this study that blazed binary diffractive elements with sampling periods approximately equal to the structural cutoff substantially outperform standard blazed échelette elements in the resonance domain. It is noteworthy that, with respect to theoretical diffraction efficiency, the comparison between blazed binary gratings and blazed gratings assumes different materials, i.e., glass for blazed échelette, and $\mathrm{TiO}_{2}$ for blazed binary gratings. A fairer comparison would assume that both gratings are fabricated of the same material. ${ }^{52}$ However, it is also worth mentioning that, because of our present fabrication constraints, blazed binary gratings etched in $\mathrm{TiO}_{2}$ do not take full advantage of the high refractive index of $\mathrm{TiO}_{2}$. For instance, while the refractive index of $\mathrm{TiO}_{2}$ is 2.3 , the maximum achievable effective index is only 1.66. The experimental and the theoretical results obtained in this study are encouraging. With the advent of deep-UV or x-ray lithography and progress in replication techniques, the mass production of low-cost highly blazed binary subwavelength diffractive elements may become possible in the near future.

\section{ACKNOWLEDGMENTS}

This work was supported by the European Community under the Reconfigurable Optical Devices for Chip Interconnects MEL-ARI program. The authors are grateful to Jean Landreau and Alain Carenco of the Centre National des Etudes de Télécommunications-Bagneux for coating the $\mathrm{TiO}_{2}$ films and to J. Frost, P. Robson, J. Woodhead, and their colleagues at the University of Sheffield (UK) for providing us with the vertical-cavity surfaceemitting laser device. They thank Jean-Claude Rodier for testing the diffractive lens and Shamlal Mallick for his careful proofreading of the manuscript. S. Astilean is with the Faculty of Physics, Department of Optics and Spectroscopy, Babes-Bolyai University, 3400 ClujNapoca, Romania. During the course of this work he was a visiting scientist at the Institut d'Optique. $\mathrm{He}$ is pleased to acknowledge the financial support provided by NATO.

\section{REFERENCES AND NOTES}

1. L. d'Auria, J. P. Huignard, A. M. Roy, and E. Spitz, "Photolithographic fabrication of thin film lenses," Opt. Commun. 5, 232-235 (1972).

2. G. J. Swanson, "Binary optics technology: the theory and design of multilevel diffractive optical elements," MIT Tech. Rep. 854 (MIT, Cambridge, Mass., 1989).

3. B. Goebel, L. L. Wang, and T. Tschudi, "Multilayer technology for diffractive optical elements," Appl. Opt. 35, 44904493 (1996).

4. J. M. Finlan, K. M. Flood, and R. J. Bojko, "Efficient $f / 1$ binary-optics microlenses in fused silica designed using vector diffraction theory," Opt. Eng. 34, 3560-3564 (1995).

5. M. T. Gale, M. Rossi, J. Pedersen, and H. Schütz, "Fabrication of continuous-relief micro-optical elements by direct laser writing in photoresists," Opt. Eng. 33, 3556-3566 (1994).

6. C. G. Blough, M. Rossi, S. K. Mack, and R. L. Michaels, "Single-point diamond turning and replication of visible and near-infrared diffractive optical elements," Appl. Opt. 36, 4648-4654 (1997).

7. F. Fujita, H. Nishihara, and J. Koyama, "Blazed gratings and Fresnel lenses fabricated by electron-beam lithography," Opt. Lett. 7, 578-580 (1982).

8. J. M. Stauffer, Y. Oppliger, P. Régnault, L. Baraldi, and M. T. Gale, "Electron beam writing of continuous resist profiles 
for optical applications," J. Vac. Sci. Technol. B 10, 25262529 (1992).

9. T. Shiono and H. Ogawa, "Diffraction-limited blazed reflection diffractive microlenses for oblique incidence fabricated by electron-beam lithography," Appl. Opt. 30, 3643-3649 (1991).

10. A. F. Harvey, Microwave Engineering (Academic, London, 1963), Sect. 13.3

11. W. E. Kock, "Metallic delay lenses," Bell Syst. Tech. J. 27, 58-82 (1948)

12. W. M. Farn, "Binary gratings with increased efficiency," Appl. Opt. 31, 4453-4458 (1992).

13. W. Stork, N. Streibl, H. Haidner, and P. Kipfer, "Artificial distributed-index media fabricated by zero-order gratings," Opt. Lett. 16, 1921-1923 (1991).

14. H. Haidner, J. T. Sheridan, and N. Streibl, "Dielectric binary blazed gratings," Appl. Opt. 32, 4276-4278 (1993).

15. Z. Zhou and T. J. Drabik, "Optimized binary, phase-only, diffractive optical element with subwavelength features for $1.55 \mu \mathrm{m}, " J$. Opt. Soc. Am. A 12, 1104-1112 (1995).

16. E. Noponen and J. Turunen, "Binary high-frequencycarrier diffractive optical elements: electromagnetic theory," J. Opt. Soc. Am. A 11, 1097-1109 (1994).

17. M. Schmitz and O. Bryngdahl, "Rigorous concept for the design of diffractive microlenses with high numerical apertures," J. Opt. Soc. Am. A 14, 901-906 (1997).

18. M. Kuittinen, J. Turunen, and P. Vahimaa, "Rigorous analysis and optimization of subwavelength-structured binary dielectric beam deflector gratings," J. Mod. Opt. 45, 133-142 (1998).

19. J. N. Mait, D. W. Prather, and M. S. Mirtoznik, "Binary subwavelength diffractive-lens design," Opt. Lett. 23, 1343-1345 (1998).

20. H. Haidner, P. Kipfer, J. T. Sheridan, J. Schwider, N. Streibl, M. Collischon, J. Hutfless, and M. Marz, "Diffraction grating with rectangular grooves exceeding $80 \%$ diffraction efficiency," Infrared Phys. 34, 467-475 (1993).

21. P. Kipfer, M. Collischon, H. Haidner, and J. Schwider, "Subwavelength structures and their use in diffractive optics," Opt. Eng. 35, 726-731 (1996).

22. M. E. Warren, R. E. Smith, G. A. Vawter, and J. R. Wendt, "High-efficiency subwavelength diffractive optical element in GaAs for 975 nm," Opt. Lett. 20, 1441-1443 (1995).

23. F. T. Chen and H. G. Craighhead, "Diffractive phase elements on two-dimensional artificial dielectrics," Opt. Lett. 20, 121-123 (1995).

24. F. T. Chen and H. G. Craighead, "Diffractive lens fabricated with mostly zeroth-order gratings," Opt. Lett. 21, 177-179 (1996).

25. J. M. Miller, N. de Beaucoudrey, P. Chavel, E. Cambril, and H. Launois, "Synthesis of subwavelength-pulse-width spatially modulated array illuminator for $0.633 \mu \mathrm{m}$," Opt. Lett. 21, 1399-1401 (1996).

26. S. Astilean, Ph. Lalanne, P. Chavel, E. Cambril, and H. Launois, "High-efficiency subwavelength diffractive element patterned in a high-refractive-index material for 633 nm," Opt. Lett. 23, 552-554 (1998)

27. Ph. Lalanne, S. Astilean, P. Chavel, E. Cambril, and H. Launois, "Blazed binary subwavelength gratings with efficiencies larger than those of conventional échelette gratings," Opt. Lett. 23, 1081-1083 (1998).

28. We believe that, with current technologies, the fabrication in glass of blazed binary diffractive elements is extremely difficult and probably impossible. Moreover, note that the use of a high-index material also has a beneficial effect on the theoretical performance (see Ref. 26).

29. M. G. Moharam, E. B. Grann, D. A. Pommet, and T. K. Gaylord, "Formulation for stable and efficient implementation of the rigorous coupled-wave analysis of binary gratings," J. Opt. Soc. Am. A 12, 1068-1076 (1995).

30. Ph. Lalanne and G. M. Morris, "Highly improved convergence of the coupled-wave method for TM polarization," J. Opt. Soc. Am. A 13, 779-784 (1996).

31. G. Granet and B. Guizal, "Efficient implementation of the coupled-wave method for metallic lamellar gratings in TM polarization,” J. Opt. Soc. Am. A 13, 1019-1023 (1996).

32. L. Li, "New formulation of the Fourier modal method for crossed surface-relief gratings," J. Opt. Soc. Am. A 14, 2758-2767 (1997).

33. Ph. Lalanne, "Effective properties and band structures of lamellar subwavelength crystals: plane-wave method revisited," Phys. Rev. B 58, 9801-9807 (1998).

34. L. Li, "Use of Fourier series in the analysis of discontinuous periodic structures," J. Opt. Soc. Am. A 13, 1870-1876 (1996).

35. D. A. Pommet, M. G. Moharam, and E. B. Grann, "Limits of scalar diffraction theory for diffractive phase elements," J. Opt. Soc. Am. A 11, 1827-1834 (1994).

36. W. Singer and H. Tiziani, "Born approximation for the nonparaxial scalar treatment of thick phase gratings," Appl. Opt. 37, 1249-1255 (1997).

37. G. J. Swanson, "Binary optics technology: theoretical limits on the diffraction efficiency of multilevel diffractive optical elements," MIT Tech. Rep. 914 (MIT, Cambridge, Mass., 1991)

38. See, for example, A. Bensoussan, J. L. Lions, and G. Papanicolaou, "Asymptotic analysis for periodic structures," in Study in Mathematics and Its Applications, J. L. Lions and G. Papanicolaou, eds. (North-Holland, Amsterdam, 1978), Chap. 4.

39. Ph. Lalanne and D. Lemercier-Lalanne, "On the effective medium theory of subwavelength periodic structures," J. Mod. Opt. 43, 2063-2085 (1996).

40. One can qualitatively understand this by considering that the pillar surrounded by the low-index material (in our case, air) may be seen as the core of a $2 \mathrm{D}$ waveguide. For a given grating period and from well-known results on 1D waveguides, multimode operations are obtained for large core widths, i.e., for large fill factors, by analogy. Conversely, for a given fill factor and a given grating period, it is intuitively clear that the structure supports an increasing number of modes for increasing values of the core refractive index.

41. H. Kikuta, Y. Ohira, H. Kubo, and K. Iwata, "Effective medium theory of two-dimensional subwavelength gratings in the non-quasi-static limit," J. Opt. Soc. Am. A 15, 1577 1585 (1998).

42. The computation was performed by the modal theory of Ref. 32 , with square truncation. Nineteen orders along each periodicity axis were retained for the computation. No convergence problems were encountered, and the numerical results can be considered as exact. These numerical results strongly differ from those obtained by Chen and Craighead (see Fig. 1 of Ref. 24). For pillar sizes of approximately $400 \mathrm{~nm}$, the zeroth-order diffraction efficiency is $65 \%$, a value $15 \%$ smaller than that reported in Ref. 24 . This difference is due to the fact that the numerical results of Ref. 24 are obtained for 16 retained orders by a slowly converging numerical method. We believe that our lowerefficiency prediction may explain the $12 \%$ discrepancy, observed by the authors of Ref. 24, between their experimental results and their numerical predictions. In our opinion, the existence of the higher-order modes is responsible for the drop in efficiency denoted by the multiplication signs; because of their oscillatory form, these modes appreciably excite the nonzero orders diffracted by the grating.

43. E. B. Grann, M. G. Moharam, and D. A. Pommet, "Artificial uniaxial and biaxial dielectrics with use of two-dimensional subwavelength binary gratings," J. Opt. Soc. Am. A 11, 2695-2703 (1994).

44. In our opinion, the pillar structure offers the advantage of open ridges that are suitable for removing material during the RIE process.

45. The depth $h$ is chosen such that a $2 \pi$-phase change occurs at normal incidence between two homogeneous thin films coated on a glass substrate and whose refractive indices are $1-\left(n_{\max }-1\right) /(2 N-2)$ and $n_{\max }+\left(n_{\max }-1\right) /(2 N-2)$. This phase change is straightforwardly obtained by use of the Airy formula [see M. Born and E. Wolf, Principles of Op- 
tics, 6th ed. (Macmillan, New York, 1964), p. 62], for homogeneous thin films. The resulting formula for $h$ is rather complex, and it turns out that, for the values of $n_{\max }$ considered hereafter, the simple expression given in Eq. (4) is valid.

46. The value of $66.5 \%$ was computed by electromagnetic theory and holds for a grating etched into glass (refractive index, $n_{g}=1.52$ ), with an optimized grating depth slightly larger than $\lambda /\left(n_{g}-1\right)$, and for unpolarized light at normal incidence from air. It is $1 \%$ larger than that obtained in Fig. 1 for a grating depth equal to $\lambda /\left(n_{g}-1\right)$.

47. Also note that the maximum pillar aspect ratio is increased from 4.6, reported in Ref. 27, to 8.8 in this study because of the use of a smaller sampling period.

48. Strictly speaking, the Airy formula for thin films has to be used, as mentioned above.
49. The diffractive components designed along the lines of procedures 1 and 2 are weakly polarization dependent. In general, we found that the first-order diffraction efficiency is a few percent larger for TE than for TM (see, e.g., Table $1)$.

50. B. Layet and M. R. Taghizadeh, "Electromagnetic analysis of fan-out gratings and diffractive lens arrays by field stitching,” J. Opt. Soc. Am. A 14, 1554-1561 (1997).

51. Y. Sheng, D. Feng, and S. Larochelle, "Analysis and synthesis of circular diffractive lens with local linear grating model and rigorous coupled-wave theory," J. Opt. Soc. Am. A 14, 1562-1568 (1997).

52. Since the width of the shadowing zone of the blazed échelette gratings decreases as the value of $n$ increases, the performance is expected to improve with the refractive index. 